\title{
AN OPTICAL AND ULTRAVIOLET SURVEY OF ALGOLS
}

\author{
JAN J. DOBIAS and MIREK J. PLAVEC \\ Department of Astronomy \\ University of California \\ Los Angeles, CA 90024-1562 \\ U.S.A. \\ (Not reviewed)
}

ABSTRACT. We present a table of results from our survey of Algois, conducted with the Lick Observatory ITS scanners and the IUE spectrometer.

We have determined the continuous flux distributions for a number of the Algol systems. Optical scans were made with the ITS scanners of Lick Observatory, while for the ultraviolet flux distributions, we used the IUE satellite spectrometer in the low-dispersion mode. The following table summarizes the results:

\begin{tabular}{lcccll}
\multicolumn{1}{c}{ Name } & phase & $\mathrm{E}(\mathrm{B}-\mathrm{V})$ & $T_{\text {eff }}$ & $\log g$ & spectrum \\
& & & & & \\
U Cephei & 0.4025 & 0.03 & 11,250 & 4.0 & B8.3 V \\
RS Cephei & 0.3212 & 0.03 & 9,700 & 3.75 & B9.7 IV-V \\
U Coronae Bor & 0.3700 & 0.03 & 14,000 & 4.0 & B6 V \\
S Equulei & 0.8552 & 0.10 & 11,250 & 4.0 & B8.3 V \\
RZ Eridani & 0.0964 & 0.03 & 7,100 & 4.25 & F0 V \\
RX Geminorum & 0.6580 & 0.03 & 9,450 & 4.0 & A0 V \\
RY Geminorum & 0.2565 & 0.04 & 9,400 & 4.0 & A0 V \\
AW Pegasi & 0.9462 & 0.00 & 9,100 & 4.0 & A1 V \\
RW Persei & 0.9011 & 0.40 & 9,750 & 3.95 & B9.6 IV-V \\
RY Persei & 0.1316 & 0.12 & $15.000::$ & & B5:: \\
U Sagittae & 0.5775 & 0.06 & 12,500 & 3.9 & B7.5 V \\
RW Tauri & 0.4645 & 0.14 & 11,750 & 4.2 & B8 V \\
S Velorum & 0.0652 & 0.04 & 8,700 & 4.0 & A2.5 V
\end{tabular}

Space Science Reviews 50 (1989), 340.

(C) 1989 by Kluwer Academic Publishers. Printed in Belgium. 